\title{
Niñas, niños, jóvenes y la etnografía: educación y descentramientos
}

\section{Children, youths and ethnography: education and de-centering}

DOI: https://doi.org/10.32870/dse.v0i20.690

\section{Diana Milstein*}

\begin{abstract}
Resumen
Este texto tiene la intención de mostrar cómo las investigaciones etnográficas, cuando incluyen la participación de niñas, niños y jóvenes, profundizan y renuevan debates existentes sobre cómo abordar, interrogar y comprender contextos educativos. Sitúo la etnografía en colaboración con niñas, niños y jóvenes como parte de una tendencia colaborativa de la etnografía educativa latinoamericana y me detengo en la actualidad de la distinción entre investigación sobre y con este sector poblacional. Además, a través del análisis de una situación tomada de una etnografía realizada en colaboración con niños y niñas, expongo oportunidades que abren esta forma compartida de investigar para acceder a comprensiones más exhaustivas e inéditas que incorporan conocimientos y teorías opacadas o desconocidas entre las y los expertos y académicos.
\end{abstract}

Palabras clave: etnografía - colaboración - niñas, niños y jóvenes - descentramientos.

\section{Resumen}

This text aims to show how ethnographic research, when it includes the participation of children and youths, delve into and renew current debates on how to address, question and understand educational contexts. I view ethnography in collaboration with children and youths as part of a collaborative trend in Latin American educational ethnography and I focus on the actuality of the distinction between research about and with this sector of the population. Moreover, through the analysis of a situation taken from ethnographic work in conducted in collaboration with children, I point to opportunities opened by this shared way to do research to access more thorough and unexplored understandings that incorporate knowledge and theories overshadowed or unknown among experts and scholars.

Palabras clave: ethnography - collaboration - children and youths - de-centering.

* Doctora en Antropología Social. Investigadora del Centro de Investigaciones Sociales (CIS)-Consejo Nacional de Investigaciones Científicas y Técnicas (CONICET)/Instituto de Desarrollo Económico y Social (IDES) y coordinadora general de la Red Internacional de Etnografía con Niños, Niñas y Jóvenes (RIENN). Ciudad Autónoma de Buenos Aires, Argentina. dianamilstein.4@gmail.com 


\section{Introducción}

Este texto tiene la intención de mostrar cómo las investigaciones etnográficas, cuando incluyen la participación de niñas, niños y jóvenes (NNJ), profundizan y renuevan debates existentes sobre la manera de abordar, interrogar y comprender contextos educativos. El punto de partida es una breve revisión de la etnografía como enfoque de investigación en contextos educativos latinoamericanos. Situar el contexto, como se verá, guarda relación no solo con las particularidades de la etnografía, sino también con los modos en que NNJ participan en los estudios. A su vez, las formas en que las y los investigadores instalan la preocupación respecto a las presencias de NNJ en los fenómenos y procesos educativos estudiados, genera abordajes que abarcan estudios sobre esta población. Esta última distinción, en sí misma, abre discusiones en el campo de la etnografía educativa, de los estudios de infancia y juventud y del propio campo de la educación. ¿Qué distingue una etnografía sobre, de una etnografía con niñas, niños y jóvenes? ¿son dos abordajes excluyentes? ¿Cómo se incorpora a NNJ en estudios de investigación? ¿La participación y colaboración de niñas, niños y jóvenes en investigaciones etnográficas procura comprender los sentidos y significados que estos sujetos otorgan a sus modos de decir, narrar y hacer? ¿busca ampliar perspectivas e interpretaciones acerca de los mundos sociales estudiados? ¿qué aportan en relación con la vida en las escuelas? ¿intentan incidir en los modos de aprender y de enseñar?

Orientada por esta forma de problematizar, el argumento que desarrollo apunta a pensar las posibilidades abiertas para explorar cómo la participación de niñas, niños y jóvenes en las etnografías enriquece maneras de interrogar, contribuye a producir conocimiento original y expande el horizonte interpretativo del campo de la investigación educativa.

\section{Etnografía, educación y participación en contextos latinoamericanos}

Los estudios etnográficos de procesos educativos tomaron impulso en Latinoamérica en la década de los ochenta del siglo XX como una alternativa claramente enmarcada dentro de las denominadas investigaciones de corte cualitativo (Anderson y Montero-Sieburth, 1998; Gomes y Gomes, 2012; Levinson, Sandoval y Bertely, 2007; Rockwell, 2001), que optimizaba la corriente cualitativa al mostrar capacidad para identificar problemas educativos asociados a reproducción social y cultural, fracaso escolar, escuelas y vida cotidiana, trabajo de las maestras, la clase y el conocimiento escolar (Nolla Cao, 1997). Asimismo, muchas de las investigaciones etnográfícas se desarrollaron en relación y/o articulación con corrientes de educación popular y movimientos políticos, sociales y culturales en lucha por demanda de derechos, recuperación de la educación pública, en oposición a toda forma de autoritarismo y en defensa de la vida democrática. Esto último orientó a una gran parte de investigaciones a estudiar contextos educativos - especialmente escolares - regionales y nacionales, y en muchos casos, a sostener vínculos estrechos con actores de las comunidades educativo-escolares.

Diálo@os sobre Educación año 11 | número 20 | enero-junio 2020 | ISSN 2007-2171 
Algunos de estos vínculos se materializaron en la colaboración de maestras, profesores e investigadores en

experiencias de coinvestigación como una modalidad de trabajo grupal en la que se combinan procesos de investigación y procesos de colectivos de coparticipación en acciones con sujetos que están involucrados en determinado problema social con el que se trabaja (Achilli, 2017: 11).

Experiencias como estas, desarrolladas por grupos en diferentes países de Latinoamérica, dejaron una huella que marcó el desarrollo de la etnografía educativa en Latinoamérica -aunque raramente es mencionada en trabajos que historizan esta corriente de investigación en educación. Tal es así, que desde mediados de las década de 1990, las experiencias de investigación etnográfica en colaboración, en nuestra región han continuado expandiéndose y profundizándose como forma de producir conocimiento sobre realidades educativas y han incorporado como colaboradores y coinvestigadores también a niñas, niños y jóvenes (Milstein, Clemente y Guerrero, 2019). Como es sabido, este impulso que dio lugar a que NNJ sean incorporados está asociado también a un contexto político, cultural y social que repercutió de manera intensa en los campos académicos de las ciencias sociales y humanas. Hago referencia a la Convención de los Derechos del Niño, de las Naciones Unidas, que provocó que la expresión "oír las voces de los niños" se convirtiera en una suerte de mantra omnipresente para activistas y gestores políticos y un símbolo de compromiso del Estado de bienestar moderno (James, 2007: 261). También, al protagonismo que han asumido niñas, niños y jóvenes en luchas políticas de distinto tipo en varios países de la región latinoamericana en las últimas décadas. Pero coincidió con la emergencia de los campos de estudio de la sociología y la antropología de la infancia y de la juventud, que estimularon estudios en los que NNJ participan como interlocutores con agencia. Este es el contexto en el que propongo pensar en la etnografía, estudiando procesos y fenómenos educativos con su participación.

Una investigación educativa etnográfica consiste en un proceso de exploración, estudio y descripción comprehensiva de fenómenos y procesos educativos realizada por uno o más investigadores con sujetos nativos para producir conocimientos nuevos; un modo de aprehender la alteridad y producir Otros para acceder a construir preguntas sobre aspectos educativos de la sociedad en estudio. Para el/la etnógrafa siempre incluye poner atención y tener bajo permanente examen los lugares y posiciones que ocupan interlocutores y etnógrafos durante los procesos de realización de las investigaciones (desde la elaboración del proyecto hasta la presentación/comunicación de resultados). Porque los lugares y posiciones que encuadran, generan y - muchas veces- definen las relaciones y los contextos de esas relaciones de nuestros interlocutores entre sí, con otros individuos y grupos y con nosotros, 
son parte imprescindible de lo que se documenta, de la construcción de datos, de los análisis y de la elaboración teórica.

\section{Actualidad de una distinción relevante: ¿sobre o con niñas, niños y jóvenes?} Investigar sobre o con niñas, niños y jóvenes es, por un lado, una manera de sintetizar un cuestionamiento a las relaciones que establecemos con quienes nos vinculamos cuando hacemos trabajo de campo, un modo de afirmar que los nativos son sujetos y no objetos de estudio. Por otro lado, condensa un cuestionamiento a abordajes de objetos genéricos e imprecisos -el niño, la niña, el joven, la joven- que esencializan, homogeneizan y neutralizan las presencias concretas y tangibles de NNJ cuyos rasgos, comportamientos, modos de comunicarse e interactuar, etc., suelen tener poca relación con las abstracciones que pretenden abarcarlos. Y también, hace referencia a polémicas referidas a las posiciones en las que se ubican las y los investigadores con respecto a sus interlocutores, tanto durante el trabajo de campo como cuando los representan en los textos. A diferencia del primer cuestionamiento aludido, en este, las y los investigadores consideran sujetos a sus interlocutores, pero definen su estudio según sea sobre infancia/s o juventud/es o niños y niñas, etc., por lo general incorporando alguna forma de participación de niñas, niños y jóvenes.

Ahora bien, la distinción entre sobre y con NNJ, con los debates implicados en la misma, no siempre se explicita en las investigaciones educativas, pero la mayor parte de estas están atravesadas — de una u otra manera - por esas discusiones. Cómo están y cómo afecta particularmente lo que hacen, dicen, sienten, piensan, conocen NNJ cuando estudiamos en contextos educativos es insoslayable. Cuando no se explicita, se amplifican y acrecientan las posibilidades de comprender los procesos investigados de maneras sesgadas y distorsionadas. Explicitar sus presencias trae consigo la posibilidad de incorporar versiones de hechos y opiniones que solo se comparten entre ellas y ellos, de volverlos visibles en discursos en los que están esencializados, de comprender cómo gravitan las vivencias personales y grupales de NNJ sobre los grandes dramas humanos y las formas en que se interrogan sobre sus significados en la circulación de conocimientos escolares (Arroyo, 2004: 115), entre otros variados aportes.

Desarrolladas brevemente estas distinciones, me detendré en la etnografía colaborativa con NNJ: "una invitación a que la construcción de conocimiento que producimos los etnógrafos en y con el campo, la realicemos de manera explícita y convenida con nuestros interlocutorescolaboradores" (Milstein, 2015a: 196).

Las investigaciones etnográficas en las que las/los investigadores referimos explícitamente la participación de NNJ durante una parte o todo el proceso de investigación, suponen su incorporación a nuestros equipos para compartir tareas vinculadas a la formulación de preguntas de investigación, al trabajo de campo o a la coautoría. De uno u otro modo, esta modalidad de la práctica etnográfica incluye una expresa e intencionada generación/producción compartida de 
conocimientos entre investigadores académicos y no académicos —en este caso, niñas, niños y jóvenes- y es presentada como parte de los resultados del proceso. Siempre teniendo en vista que este compartir, en ningún caso queda reducido a un rasgo metodológico ni restringe temáticas de investigación a tópicos asociados a la niñez, la infancia y la juventud. Muy por el contrario, conduce a profundizar en los problemas y comprensiones sobre variadas y amplias temáticas de la vida social. ${ }^{1}$

\section{Etnografía, colaboración y descentramientos}

Las y los investigadores que realizamos etnografías como parte constante de nuestra actividad, practicamos distintas modalidades de descentramientos. Buscamos corrernos de los ejes que centran nuestras visiones y organizan nuestras emociones, percepciones y conocimientos. Trabajamos con nosotros mismos para producirnos desplazamientos cognitivos que nos permitan distinguir "centrismos" incorporados — derivados de las identidades que nos constituyen como parte de una sociedad marcadamente desigual — según clase social, raza, género, nacionalidad, edad, generación, religión, tradición académica. Para eso, una de las estrategias que aprendemos a usar son las relaciones de alteridad, básicas e imprescindibles en nuestras formas de interrogación empírico-teórica. De ahí que la cuestión de la otredad vertebra y organiza el curso de nuestras investigaciones, teniendo en cuenta que

el otro no se define como fundamentalmente aparte; el otro se suma al "nosotros" en la perplejidad de compartir una misma temporalidad, un mismo espacio y una misma coevalness (Fabian 1983) [...] englobando al propio antropólogo en su relación con la alteridad (Peirano, 1995: 109).

Hacer etnografías educativas con la colaboración de NNJ potencia las posibilidades de ese descentramiento necesario y, con ello, también abre oportunidades para descentrar temáticas, es decir, alejarse, tomar distancia de temas que se constituyen como centrales y predefinen lo que se investiga y cómo se investiga en el campo de la educación. Un ejemplo² nos permitirá explicar más acabadamente este último descentramiento al que estoy aludiendo.

En el año 2005 realicé trabajo de campo en Villa La Florida, Quilmes, una localidad ubicada a 25 kilómetros al sur de la ciudad de Buenos Aires (Argentina), como parte de una investigación que buscaba "reconocer las presencias de prácticas políticas al interior de las escuelas como expresiones de campo político extraescolar" (Milstein, 2009: 17). Entre septiembre y diciembre, cinco niñas y un niño que tenían entre 11 y 14 años, alumnas de una de las escuelas primarias de

1 Para conocer más sobre esta amplitud en estudios realizados en la región latinoamericana, puede consultarse el texto de J. Jaramillo y S. Fernández (2018).

2 La situación que aquí presento como evidencia etnográfica está publicada con variantes en un capítulo en coaturía (Milstein, Clemente y Guerrero, 2018). 
la localidad, hicieron trabajo de campo conmigo. El propósito del trabajo del equipo que conformamos con ellas era conocer y registrar versiones acerca de la vida en la localidad y la escuela, tomando como punto de partida la percepción que tenían del mundo social dominado por el sentir, decir y hacer de los adultos. ${ }^{3}$ Este trabajo compartido con las niñas resultó crucial para comprender que esas presencias de prácticas políticas que me asombraban, eran una evidencia del giro político que se ha producido en las escuelas públicas estatales argentinas durante las últimas décadas.

Algunas actividades de nuestro trabajo de campo las hacíamos en grupo y otras de manera individual y luego se compartían los registros fotográficos y de audio ${ }^{4}$ en reuniones de equipo -en muy pocas oportunidades los niños realizaron registros escritos, mientras que yo siempre compartía mis notas de campo escritas. Para documentar por escrito las vivencias y conversaciones, yo transcribía la grabación de las actividades de campo y de nuestras reuniones, luego leíamos estos documentos con minuciosidad y tomaba también mis notas. Las niñas leían parte de estos documentos guiadas por el interés del momento, comentaban alguna frase, generaban asociaciones con otras historias, relataban alguna anécdota ya escrita agregando detalles, entre otras tantas modalidades que iban surgiendo y que, en algunos casos, resultaron el inicio de un camino reflexivo compartido, interesante para el estudio que hacíamos en conjunto y para mi propio trabajo de investigación. Así sucedió un día que escuchamos el audio de la entrevista que una niña le había hecho a su tía Ana. Al finalizar se produjo el siguiente intercambio:

—Tiene suerte. Trabaja en la política — dijo Patricia.

- ¿Por qué? - pregunté.

-Y está siempre en la oficina — dijo Camila sonriendo.

-Con papeles, habla por teléfono, recibe - comentó Leonor.

— ¿A quién recibe? —pregunté.

-Da la leche a los que hacen fila y otras cosas y van y conversan. A veces los tiene que sacar porque se llena la oficina y no puede con tantos. La otra vez dicen que fue Cristina ${ }^{5}$ -dijo Leonor.

Este diálogo me dejó pensando en la importancia que tenía esta actividad. Para estas niñas era uno de los trabajos en los que se empleaban quienes vivían en la localidad. Yo sabía que Villa La Florida era uno de los lugares donde más claramente podían advertirse los efectos de las políticas económicas y sociales implementadas a lo largo de la década de los noventa del

3 Acerca de esta experiencia de etnografía en colaboración con niños puede consultarse Milstein (2008, 2009, 2010a, 2010b, 2011, 2015a, 2015b).

4 Utilizamos cámaras fotográficas analógicas con lente básica y grabadores con casetes.

5 Se referían a Cristina Kirchner, candidata a senadora en las elecciones de octubre de 2005. 
siglo XX. Una gran parte de hombres y mujeres eran obreros de fábricas grandes e importantes que cerraron o redujeron su actividad de manera considerable y en ocho años produjeron alrededor de $40 \%$ de vecinos desocupados. Esta situación, junto con un proceso visible de desestructuración y desorganización del Estado nacional y de los estados provinciales, condujo a un proceso de protesta social en las calles que culminó en 2001 con una pueblada ${ }^{6}$ que se manifestó en forma local y atravesó las vidas cotidianas de las familias de trabajadores desocupados en Villa La Florida. Asimismo, muchas escuelas públicas fueron lugares donde grupos familiares organizaban actividades comunitarias: ollas populares, intercambio de ropa, espacios para dormir y estar para quienes perdían sus viviendas, etc. Al mismo tiempo, en muchas localidades como en la que estaba realizando esta investigación, se expandían redes clientelares lideradas por caudillos y punteros de los dos partidos políticos mayoritarios que en los primeros años del siglo XXI se consolidaron, a pesar del recelo y el rechazo mayoritario hacia el mundo de "la política" y "los políticos", expresado en la pueblada de 2001.

Cuando escuché la entrevista a la tía, contando que trabajaba en la política, y participé del diálogo con las niñas que se produjo posteriormente, reparé en la dimensión que tenía en la vida cotidiana de las familias esta forma novedosa de empleo en la política, en una comunidad cuya actividad económica mayoritaria estaba vinculada a fábricas de vidrio, textiles, papeleras, dulceras y al pequeño comercio.

Las redes clientelares eran comprendidas por los estudios antropológicos y sociológicos más actuales como parte de las respuestas organizadas por el Estado para generar contención social y ejercer control ante la desocupación y la protesta social, así como para alimentar una estructura política a la que denominaban intermedia (Auyero, 2001; Svampa y Pereyra, 2003). Sin embargo, no me parecía que podía pensar lo de la tía Ana con esta interpretación.

Unos días después de aquel diálogo, nos reunimos nuevamente con el grupo en un parque. La actividad era contar historias. En un momento dado, pasó por la avenida aledaña un auto desde el que alguien arengaba a la gente a votar por su candidato.

Uno de los chicos comenzó a imitar el cántico que escuchaba, nos reímos mucho y una de las niñas dijo: "Debe ser Ana, tu tía”.

Yo miré a la sobrina y ella dijo:

Viste, mi tía, la que le dio las cosas fue Cristina, y le dijo que se las daba para que la voten. Porque mi tía está en ese plan porque necesita una vivienda porque son nueve chicos y no puede con todo. Está en un plan y la que le dio la mercadería le dijo que ella tenía que votar a Cristina, mi tía contó. Después ya le dieron la oficina que es mejor trabajo.

6 En Argentina se utiliza pueblada para denominar levantamientos y revueltas populares en los que miles de personas salen a protestar a las calles. 
La conversación continuó con comentarios acerca de los trabajos de los padres y una de las niñas dijo:

A mí la política no me importa porque soy extranjera y no podemos votar y a mi mami nunca le van a dar un trabajo así porque ella es paraguaya y trabaja limpiando, no vuelve a mi casa porque se queda a dormir en el trabajo.

Retomé mis notas intentando incorporar este modo de ver la política como un trabajo, pero no vinculada a observar cambios en la estructura política. Para estos niños y sus familias, la política era una posibilidad de ascenso frente a trabajos como el empleo en casas de familias para limpieza general o el cartoneo. Era el salario lo que ellos miraban, era sus vidas familiares y las de niñas y niños como ellos. Era la política como parte del cotidiano en este mundo social donde las fábricas no estaban. Escribí entonces que con estas niñas había podido alcanzar una comprensión más acertada que rompía con la idea de que la política se reducía a ámbitos de la vida social; más bien había que estudiarla en el comportamiento de quienes se supone que no tienen condiciones ni atributos para desarrollarla. Así, tuve la posibilidad de comprender cabalmente hasta qué punto también lugares como la escuela podían ser estudiados incorporando la política como una dimensión que develaba relaciones, disputas y tensiones que desmentían la neutralidad de las escuelas. Las escuelas había que entenderlas como lugares atravesados por la política y lo político.

\section{A modo de cierre}

En este trabajo presté atención a algunos de los efectos que resultan del trabajo compartido entre investigadores y niñas, niños y jóvenes cuando estos últimos intervienen activamente y de manera expresa en procesos de producción de conocimiento que supone una etnografía educativa.

Comencé reseñando cómo incorporar a niñas, niños y jóvenes en la etnografía educativa latinoamericana; ingresó enriqueciendo una vertiente colaborativa que se venía experimentando con buenos resultados. Se trataba de integrar intelectuales no académicos, como los docentes, al proceso etnográfico de producción de conocimiento educativo. La colaboración de NNJ - que no son trabajadores intelectuales_ con etnógrafos fluyó en esta vertiente y produjo experiencias que, en conjunto, llevan más de dos décadas.

Intenté mostrar lo que estas han aportado en relación con dos temas en discusión que inciden en el campo de la investigación educativa y el de los estudios sobre infancias y juventudes, así como otros sobre los que no me detengo en este trabajo.

Uno de estos temas trata acerca de la participación en investigaciones y se suele sintetizar como disyuntiva: con o sobre niñas, niños y jóvenes. Es un debate relevante en tanto exige

Diólo@os

sobre Educación

TEMAS ACTUALES EN INVESTIGACTON EDCA año 11 | número 20 | enero-junio 2020 | ISSN 2007-2171 
exponer concepciones acerca de infancia/niñez/juventud, que afectan de manera directa las posibilidades o no de incorporar NNJ como interlocutores y/o co-investigadores, reduce o amplía los temas y problemas que pueden abordarse de manera compartida entre investigadores y NNJ, y altera las posiciones y roles de niños, jóvenes y adultos — no solo investigadores - en las relaciones sociales.

El otro se refiere a los descentramientos. Dentro de este amplísimo debate consideré a el/ la investigadora y a los temas/problemas de investigación tal como se constituyen en el campo de la educación. Señalé que una faceta se vincula a que es condición y necesidad para las y los etnógrafos, desplazar-nos de modos de percibir, sentir, actuar y pensar para poder producir alteridades. La otra faceta la relacioné con un replanteo del problema de investigación. Mostré, a través de la descripción de una situación vivida con niñas con quienes realizaba trabajo de campo, cómo quedó modificada de manera irreversible un aspecto del abordaje de la política y lo político en un contexto escolar.

Así, realizar etnografía en colaboración con niñas, niños y jóvenes abre oportunidades en el campo de la investigación educativa para trasformar sujetos, tanto adultos como NNJ, quienes, luego de formar parte de un proceso de estudio etnográfico, experimentan trasformaciones que modifican sus modos de ser y estar en los mundos sociales. Asimismo, acentúa las posibilidades que tiene el enfoque etnográfico para apartarse de problemas y temas educativos encuadrados en términos normativo-prescriptivos, y así acceder a comprensiones más exhaustivas e inéditas que incorporan conocimientos y teorías opacadas o desconocidas entre los y las expertas y académicos.

\section{Referencias}

Achilli, E. (2017) Construcción de conocimientos antropológicos y coinvestigación etnográfica. Cuadernos de Antropología Social, (45), 7-20.

Anderson, G. y Montero-Sieburth, M. (1998). Educational Qualitative Research in Latin America, The Struggle for a New Paradigm. Nueva York: Garland Publishing.

Arroyo, M. (2004). Imagens quebradas. Petropolis: Vozes.

Auyero, J. (2002). Clientelismo político en Argentina: doble vida y negación colectiva. Perfiles Latinoamericanos, México: FLACSO, (20), 33-52.

Fabian, J. 1983. Time and the other. Columbia: Columbia University Press.

Gomes, A. M. R. y N. L. Gomes (2011). Anthropology and education in Brazil: possible pathways. In: Anderson-Levitt (ed.). Anthropologies of education. A global guide to ethnographic studies of learning and schooling (pp.11-131). Nueva York: Berghahn Books.

James, A. (2007). Giving Voice to Children's Voices: Practices and Problems, Pitfalls and Potentials. American Anthropologist, 109(2), 261-272. 
Jaramillo, J. y S. Fernández (eds.) (2018). Panorama sobre etnografía con niños, niñas, adolescentes y jóvenes en Argentina, Brasil, Colombia y Ecuador: 1995- 2016. Argentina: RIENN. Libro digital. Levinson, B. A. U., E. Sandoval-Flores y M. Bertely-Busquets (2007). Etnografía de la educación. Tendencias actuales. Revista Mexicana de Investigación Educativa. http://www.redalyc.org/ articulo.oa?id=14003402

Milstein, D. (2008). Conversaciones y percepciones de niños y niñas en las narrativas antropológicas. Sociedade e Cultura, 11(1). https://doi.org/10.5216/sec.v11i1.4470 (2009). La nación en la escuela.Viejas y nuevas tensiones políticas. Buenos Aires: Miño y Dávila/CAS/IDES.

(2010a). Children as Co-researchers in Anthropological Narratives in Education. Ethnography and Education, 5(1), 1-15.

(2010b). Politics is also Child's Play. Teaching and Teacher Education 26, 136-143.

(2011). Encuentros etnográficos con niñ@s. Campo y reflexividad. En Milstein, D., A. Clemente, M. Dantas, A. Guerrero y M. J. Higgins (eds.).Encuentros etnográficos con niñ@s y adolescentes. Entre tiempos y espacios compartidos (pp. 217-236). Buenos Aires: Miño y Dávila/ IDES/CAS.

(2015a). Etnografía con niños y niñas: oportunidades educativas para investigadores. Espacios en Blanco. Revista de Educación, (25), 193-211.

(2015b). Constructing Collaborative Interpretations: Children as Cresearchers in an Ethnographic Study in Argentina. En P. Smeyers, D. Bridges, N. Burbules y M. Griffiths (eds.), International Handbook of Interpretation in Educational Research (pp. 529-549). Dordrecht: Springer International Handbooks of Education.

A. Clemente y A. Guerrero (2018). Writing as New Understandings of Social Phenomena: The Practice of Including Children's Perspectives. En Jeffrey, B. y L. Russel (eds.). Ethnographic writing.New Cottage: E\&E Publishers, 139-154.

(2019. Collaboration in Educational Ethongraphy in Latin America. Oxford Research Encyclopedias Education. https://oxfordre.com/education/view/10.1093/acrefore/9780190264093.001.0001/acrefore-9780190264093-e-565

Nolla Cao, N. (1997). Etnografía, una alternativa más en la investigacion pedagógíca, Educación Médica Superior, (11), 2. Habana: Ministerio de Salud Pública, 107-115.

Peirano, M. (1995). A favor da Etnografía. Río de Janeiro: Relume-Dumará. http://www.marizapeirano.com.br/livros/a favor da etnografia.pdf

Rockwell, E. (2001). Caminos y rumbos de la investigación etnográfica en América Latina. Cuadernos de Antropología Social, (13), 53-64.

Svampa, M. y S. Pereyra (2003). Entre la ruta y el barrio. La experiencia de las organizaciones piqueteras. Buenos Aires: Biblos. 